Supplement of Solid Earth, 9, 1141-1156, 2018

https://doi.org/10.5194/se-9-1141-2018-supplement

(C) Author(s) 2018. This work is distributed under

the Creative Commons Attribution 4.0 License.

(c) (1)

Supplement of

\title{
Multiscale porosity changes along the pro- and retrograde deformation path: an example from Alpine slates
}

Ismay Vénice Akker et al.

Correspondence to: Ismay Vénice Akker (ismay.akker@geo.unibe.ch)

The copyright of individual parts of the supplement might differ from the CC BY 4.0 License. 


\begin{tabular}{|c|c|c|c|c|c|c|c|c|c|c|c|}
\hline Helium pycnometry & Sample & Comment & $\begin{array}{c}\text { Geometrical } \\
\text { volume } \\
(\mathrm{cm} 3) \\
\end{array}$ & Mass (g) & $\begin{array}{c}\text { Bulk } \\
\text { density } \\
(\mathrm{g} / \mathrm{cm} 3) \\
\end{array}$ & $\begin{array}{c}\text { grain } \\
\text { volume } \\
(\mathrm{cm} 3) \\
\end{array}$ & $\begin{array}{c}\text { stdev. } \\
\text { grain } \\
\text { volume } \\
(\mathrm{cm3}) \\
\end{array}$ & $\begin{array}{c}\text { grain } \\
\text { density } \\
(\text { g/cm3) } \\
\end{array}$ & $\begin{array}{l}\text { stdev. } \\
\text { grain } \\
\text { density } \\
(\mathrm{g} / \mathrm{cm} 3) \\
\end{array}$ & Porosity (vol\%) & $\begin{array}{c}\text { stdev. } \\
\text { Porosity } \\
\text { (vol\%) } \\
\end{array}$ \\
\hline \multirow{6}{*}{$\begin{array}{c}\text { total of layers } \\
\text { average separate } \\
\text { layers }\end{array}$} & $B \# 1$ & & 4.48 & 11.97 & 2.67 & 4.32 & 0.00 & 2.77 & 0.00 & 3.65 & 0.00 \\
\hline & $B \# 2$ & & 4.17 & 11.20 & 2.69 & 4.05 & 0.00 & 2.77 & 0.00 & 2.96 & 0.00 \\
\hline & B total & & 8.65 & 23.16 & 2.68 & 8.37 & 0.00 & 2.77 & 0.00 & 3.32 & 0.00 \\
\hline & $B$ & & 4.33 & 11.58 & 2.68 & 4.18 & 0.00 & 2.77 & 0.00 & 3.31 & 0.00 \\
\hline & $C \# 1$ & & 4.88 & 12.59 & 2.58 & 4.55 & 0.00 & 2.76 & 0.00 & 6.65 & 0.00 \\
\hline & $C \# 2$ & & 4.86 & 12.39 & 2.55 & 4.48 & 0.01 & 2.77 & 0.00 & 7.82 & 0.00 \\
\hline \multirow{4}{*}{$\begin{array}{c}\text { total of layers } \\
\text { average separate } \\
\text { layers }\end{array}$} & C total & & 9.74 & 24.98 & 2.56 & 9.05 & 0.00 & 2.76 & 0.00 & 7.03 & 0.00 \\
\hline & $C$ & & 4.87 & 12.49 & 2.56 & 4.52 & 0.01 & 2.76 & 0.00 & 7.24 & 0.00 \\
\hline & $D \# 1$ & & 13.81 & 37.75 & 2.73 & 13.72 & 0.01 & 2.75 & 0.00 & 0.67 & 0.00 \\
\hline & $D \# 2$ & & 12.38 & 33.75 & 2.73 & 12.30 & 0.01 & 2.74 & 0.00 & 0.65 & 0.00 \\
\hline \multicolumn{12}{|l|}{ average separate } \\
\hline \multirow[t]{3}{*}{ layers } & $D$ & & 13.10 & 35.75 & 2.73 & 13.01 & 0.01 & 2.75 & 0.00 & 0.66 & 0.00 \\
\hline & $E \# 1$ & & 3.99 & 10.78 & 2.70 & 3.90 & 0.00 & 2.77 & 0.00 & 2.27 & 0.00 \\
\hline & $E \# 2$ & & 5.09 & 13.72 & 2.69 & 4.95 & 0.00 & 2.77 & 0.00 & 2.85 & 0.00 \\
\hline \multirow{4}{*}{$\begin{array}{c}\text { total of layers } \\
\text { average separate } \\
\text { layers }\end{array}$} & E total & & 9.08 & 24.50 & 2.70 & 8.83 & 0.00 & 2.78 & 0.00 & 2.77 & 0.00 \\
\hline & $E$ & & 4.54 & 12.25 & 2.70 & 4.42 & 0.00 & 2.77 & 0.00 & 2.56 & 0.00 \\
\hline & $F \# 1$ & & 10.24 & 27.04 & 2.64 & 10.02 & 0.00 & 2.70 & 0.00 & 2.11 & 0.00 \\
\hline & $F \# 2$ & & 9.54 & 25.38 & 2.66 & 9.43 & 0.00 & 2.69 & 0.00 & 1.13 & 0.00 \\
\hline \multicolumn{12}{|l|}{ average separate } \\
\hline layers & $F$ & & 9.89 & 26.21 & 2.65 & 9.73 & 0.00 & 2.69 & 0.00 & 1.62 & 0.00 \\
\hline & $G \# 1$ & $<10 \%$ chamber fill & 3.45 & 9.22 & 2.67 & 3.33 & 0.00 & 2.77 & 0.00 & 3.51 & 0.00 \\
\hline & $G \# 2$ & $<10 \%$ chamber fill & 2.30 & 6.17 & 2.68 & 2.24 & 0.00 & 2.76 & 0.00 & 2.98 & 0.00 \\
\hline & $G \# 3$ & $<10 \%$ chamber fill & 1.43 & 3.80 & 2.67 & 1.38 & 0.00 & 2.75 & 0.00 & 3.15 & 0.01 \\
\hline & $G \# 4$ & $<10 \%$ chamber fill & 1.47 & 3.93 & 2.67 & 1.42 & 0.00 & 2.76 & 0.00 & 3.20 & 0.01 \\
\hline & $G \# 5$ & $<10 \%$ chamber fill & 1.62 & 4.20 & 2.59 & 1.52 & 0.00 & 2.76 & 0.00 & 5.99 & 0.01 \\
\hline & $G \# 5$ & duplo measurement & 1.62 & 4.19 & 2.59 & 1.52 & 0.00 & 2.76 & 0.00 & 6.06 & 0.01 \\
\hline & $G \# 6$ & $<10 \%$ chamber fill & 1.48 & 3.91 & 2.65 & 1.42 & 0.00 & 2.75 & 0.00 & 3.73 & 0.01 \\
\hline
\end{tabular}




\begin{tabular}{|c|c|c|c|c|c|c|c|c|c|c|c|}
\hline & $G \# 7$ & $<10 \%$ chamber fill & 0.94 & 2.46 & 2.62 & 0.90 & 0.00 & 2.75 & 0.00 & 4.57 & 0.01 \\
\hline \multirow{5}{*}{$\begin{array}{l}\text { total of layers } \\
\text { average separate } \\
\text { layers }\end{array}$} & G total & & 12.68 & 33.69 & 2.66 & 12.15 & 0.00 & 2.77 & 0.00 & 4.23 & 0.00 \\
\hline & $G$ & & 1.81 & 4.81 & 2.65 & 1.74 & 0.00 & 2.76 & 0.00 & 4.15 & 0.01 \\
\hline & Gl \#A & $<10 \%$ chamber fill & 1.39 & 3.65 & 2.62 & 1.32 & 0.00 & 2.76 & 0.00 & 5.18 & 0.01 \\
\hline & $G 1 \# B$ & $<10 \%$ chamber fill & 2.85 & 7.72 & 2.71 & 2.81 & 0.00 & 2.75 & 0.00 & 1.42 & 0.01 \\
\hline & $G 1 \# C$ & $<10 \%$ chamber fill & 1.14 & 3.03 & 2.65 & 1.10 & 0.00 & 2.76 & 0.00 & 3.80 & 0.00 \\
\hline \multirow{7}{*}{$\begin{array}{l}\text { total of layers } \\
\text { average separate } \\
\text { layers }\end{array}$} & G1 total & $<10 \%$ chamber fill & 5.39 & 14.41 & 2.67 & 5.23 & 0.00 & 2.76 & 0.00 & 3.04 & 0.00 \\
\hline & G1 & $<10 \%$ chamber fill & 1.80 & 4.80 & 2.66 & 1.74 & 0.00 & 2.76 & 0.00 & 3.47 & 0.01 \\
\hline & $G 2 \# A$ & $<10 \%$ chamber fill & 1.00 & 2.66 & 2.67 & 0.97 & 0.00 & 2.75 & 0.01 & 2.79 & 0.01 \\
\hline & $G 2 \# B$ & $<10 \%$ chamber fill & 0.65 & 1.70 & 2.60 & 0.62 & 0.00 & 2.74 & 0.00 & 5.16 & 0.02 \\
\hline & $G 2 \# C$ & $<10 \%$ chamber fill & 2.20 & 5.89 & 2.68 & 2.15 & 0.00 & 2.75 & 0.00 & 2.24 & 0.00 \\
\hline & $G 2 \# D$ & $<10 \%$ chamber fill & 0.39 & 0.99 & 2.57 & 0.36 & 0.00 & 2.74 & 0.01 & 6.29 & 0.03 \\
\hline & $G 2 \# E$ & $<10 \%$ chamber fill & 0.83 & 2.19 & 2.65 & 0.79 & 0.00 & 2.76 & 0.01 & 4.14 & 0.01 \\
\hline \multirow{4}{*}{$\begin{array}{l}\text { total of layers } \\
\text { average separate } \\
\text { layers }\end{array}$} & G2 total & & 5.06 & 13.43 & 2.66 & 4.87 & 0.00 & 2.76 & 0.00 & 3.62 & 0.00 \\
\hline & G2 & & 1.01 & 2.69 & 2.63 & 0.98 & 0.00 & 2.75 & 0.00 & 4.12 & 0.01 \\
\hline & $H \# 1$ & & 7.34 & 19.54 & 2.66 & 7.14 & 0.00 & 2.74 & 0.00 & 2.74 & 0.00 \\
\hline & $H \# 2$ & & 5.93 & 15.91 & 2.68 & 5.82 & 0.00 & 2.73 & 0.00 & 1.81 & 0.00 \\
\hline \multirow{2}{*}{$\begin{array}{l}\text { total of layers } \\
\text { average separate } \\
\text { layers }\end{array}$} & H total & & 13.27 & 35.45 & 2.67 & 12.96 & 0.00 & 2.74 & 0.00 & 2.40 & 0.00 \\
\hline & $H$ & & 6.64 & 17.73 & 2.67 & 6.48 & 0.00 & 2.73 & 0.00 & 2.28 & 0.00 \\
\hline
\end{tabular}

Table S1. Overview of all He-pycnometry data. 


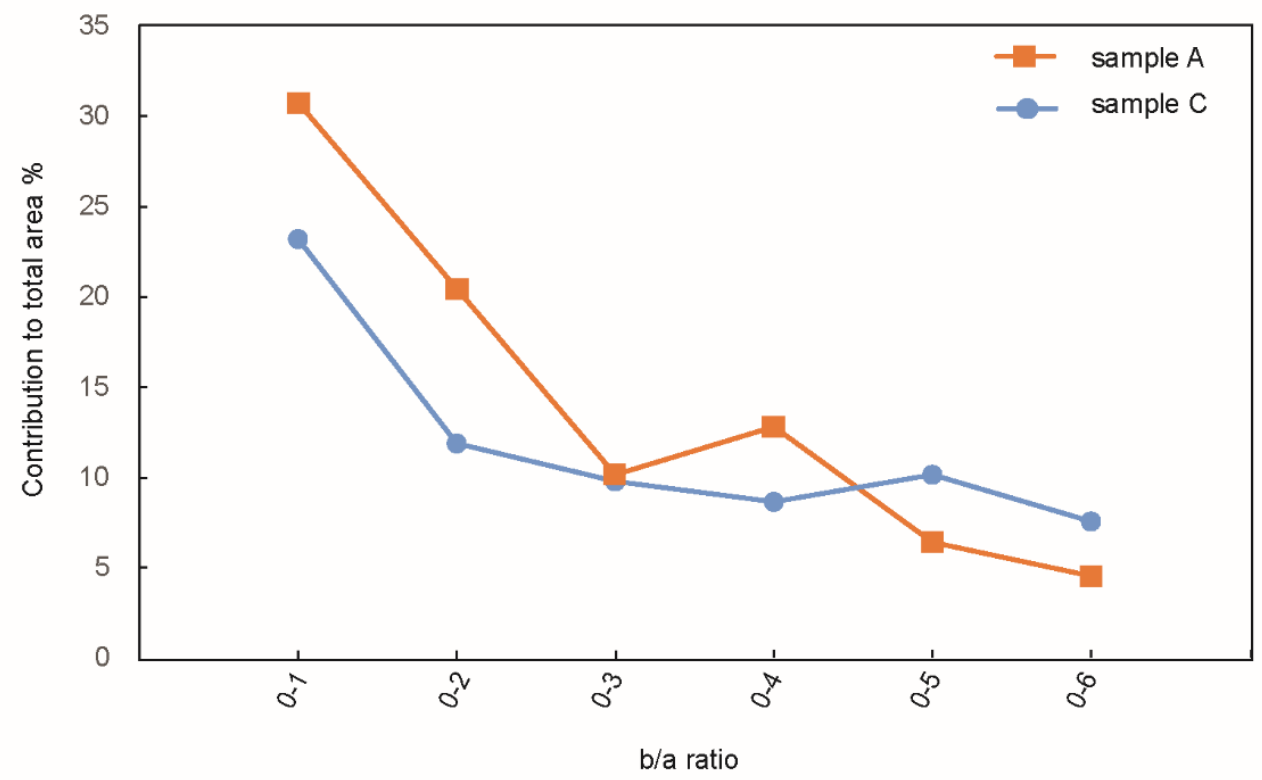

Figure S1. Plot of minor/major axis (b/a ratio) vs. the area $\%$ shows that fractures are defined from a ratio of $<0.3$. To be sure that all pores are microfractures a ratio of $<0.2$ is chosen. 


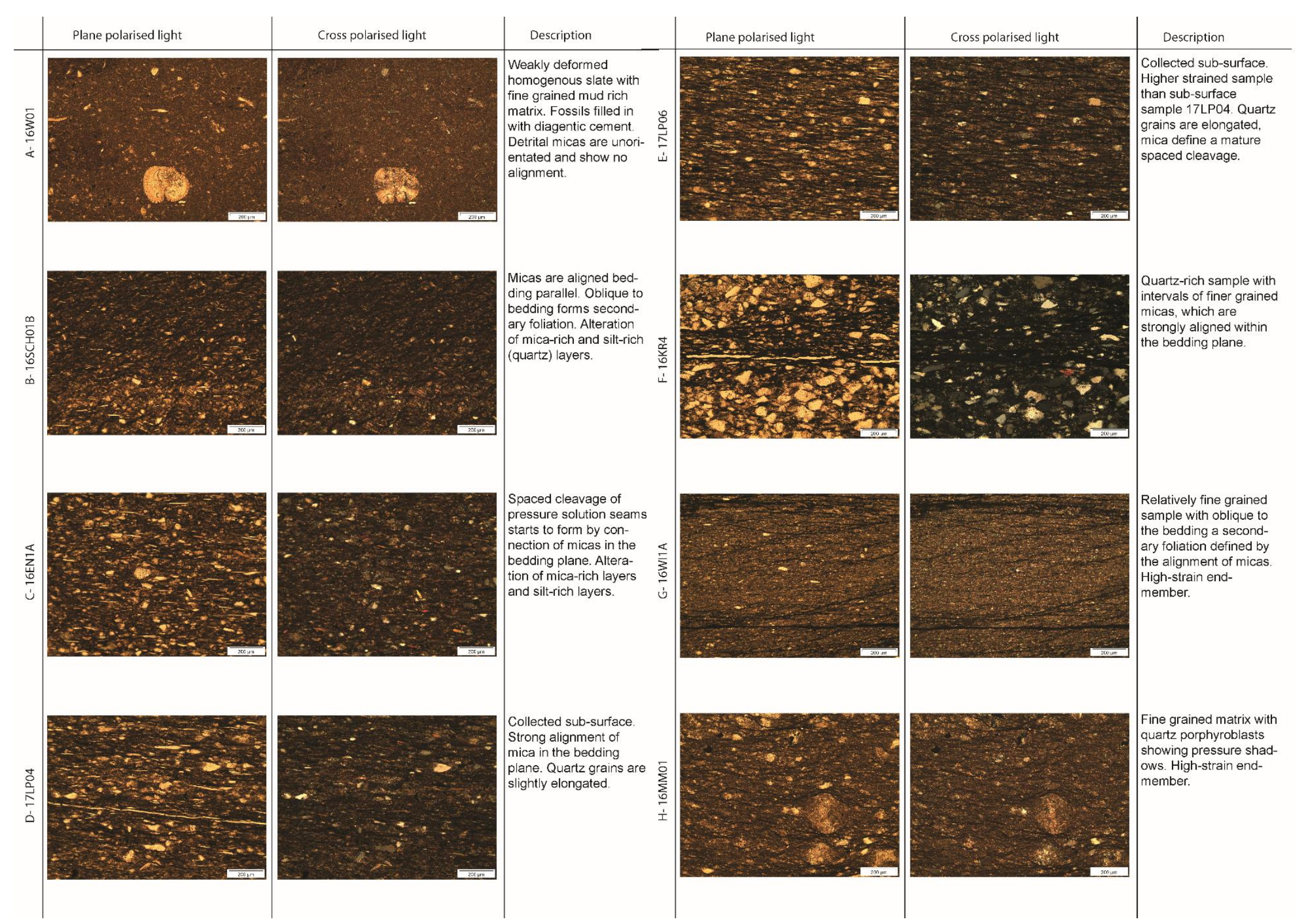

Figure S2. Photomicrographs from thin sections in both plane and cross-polarised light give an overview of microstructures from all used samples in this study. 

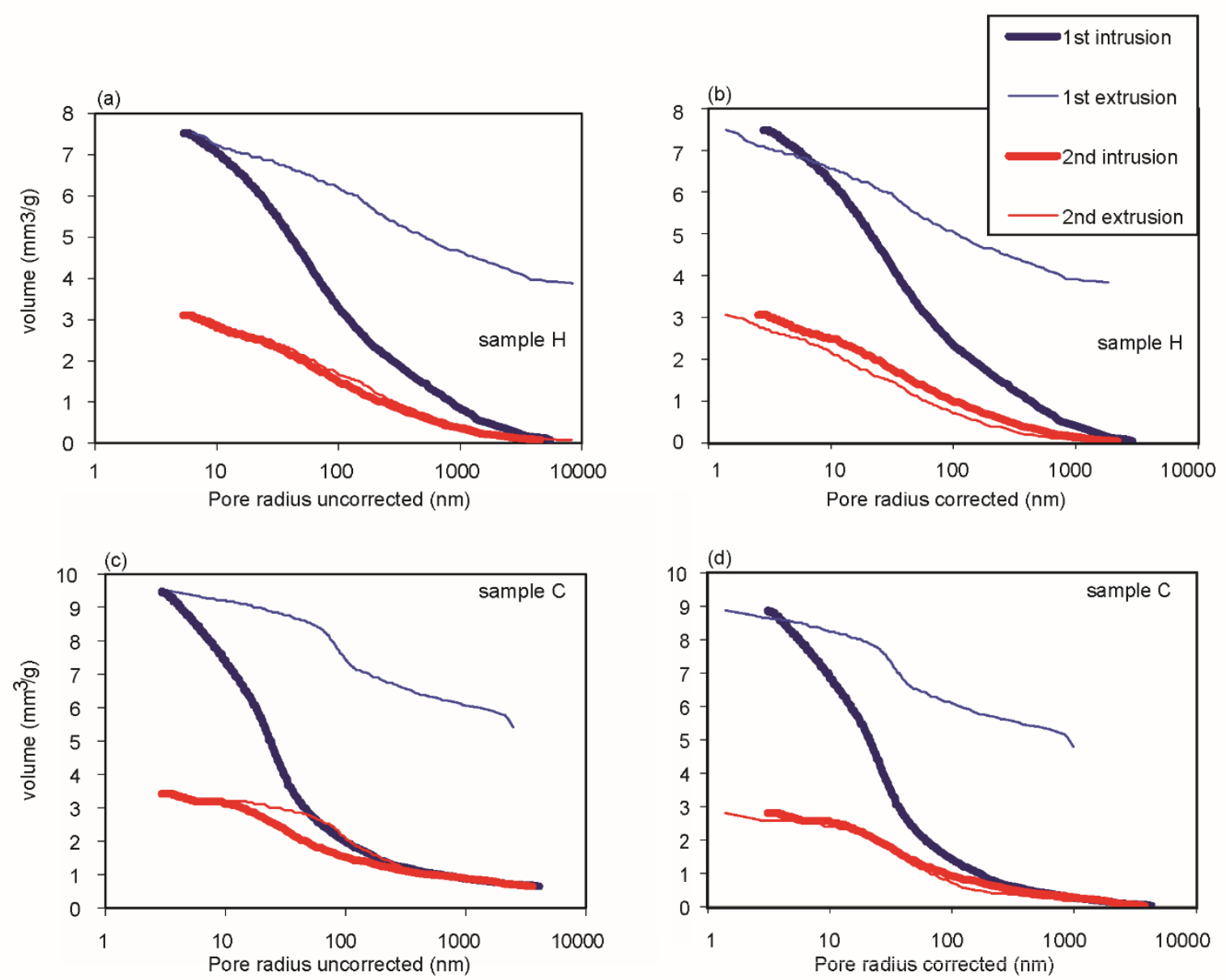

Figure S3. Mercury Intrusion Porosimetry data showing $1^{\text {st }}$ and $2^{\text {nd }}$ intrusion and extrusion curve uncorrected and corrected for the contact angle in the extrusion data. $\mathrm{a}-\mathrm{b})$ sample $\mathrm{H}$ shows that in the corrected data the hysteresis between the $2^{\text {nd }}$ intrusion and extrusion cycle slightly increases. $\left.c-d\right)$ sample $C$ shows that in the corrected data the hysteresis between the $2^{\text {nd }}$ intrusion and extrusion cycle reduces. 